\title{
Incidence and mortality of patients transported by ambulance during the first surge of the COVID-19 pandemic in Osaka Prefecture, Japan
}

Yusuke Katayama ( $\sim$ orion13@hp-emerg.med.osaka-u.ac.jp )

Osaka University

Kenta Tanaka

Osaka University

Tetsuhisa Kitamura

Osaka University

Taro Takeuchi

Osaka University

Shota Nakao

Rinku General Medical Center

Masahiko Nitta

Osaka Medical College

Taku Iwami

Kyoto University

Satoshi Fujimi

Osaka Prefectural Medical Center

Toshifumi Uejima

Kindai University

Yuuji Miyamoto

Otemae Hospital

Takehiko Baba

Baba Memorial Hospital

Yasumitsu Mizobata

Osaka City University

Yasuyuki Kuwagata

Kansai Medical University

Takeshi Shimazu

Osaka University

\section{Tetsuya Matsuoka}

Rinku General Medical Center 


\section{Research Article}

Keywords: COVID-19, Coronavirus, emergency medical service (EMS) system

Posted Date: February 25th, 2021

DOl: https://doi.org/10.21203/rs.3.rs-209325/v1

License: (c) (i) This work is licensed under a Creative Commons Attribution 4.0 International License. Read Full License 


\section{Abstract}

Background; Novel corona virus (COVID-19) outbreaks have spread worldwide. Although the COVID-19 pandemic affects the emergency medical service (EMS) system, which is one factor of primary care, little is known about its impact. This study aimed to reveal the impact of the COVID-19 pandemic on the EMS system and outcome of patients transported by ambulance.

Methods; This was a retrospective study with a study period from 1 January 2020 to 31 May 2020. We included patients transported by ambulance who were registered in a population-based registry of patients transported by ambulance. The endpoints of this study were the incident number of patients transported by ambulance each month and the number of deaths among these patients admitted to hospital each month. We calculated the incidence rate ratio (IRR) and $95 \%$ confidence interval (CI) using a Poisson regression model with year 2019 as the reference.

Results; From January to May 2019, 205,195 patients were transported, whereas from January to May $2020,180,362$ patients were transported, indicating a significant decrease in the number of emergency patients transported by ambulance (IRR: $0.88,95 \% \mathrm{Cl}$ : 0.87-0.88). The number of deaths among emergency patients admitted to hospital was 5237 in January-May 2019 and remained unchanged at 5172 in January-May 2020 (IRR: 0.99, 95\% Cl: 0.95-1.03).

Conclusion; The first surge of the COVID-19 pandemic had no adverse effect on the EMS system in Osaka Prefecture, Japan.

\section{Introduction}

Outbreaks of infection by novel corona virus (COVID-19), which was confirmed in Wuhan, China in December 2019, have spread not only in China but also around the world. In Japan, the number of patients with COVID-19 exceeded 120,000 on 19 November 2020.[1] The characteristics of COVID-19 are that some of its symptoms, such as fever, cough, sore throat, and general malaise, are common with other upper respiratory tract infections, and some patients are asymptomatic.[2] However, severely affected patients, which account for approximately $20 \%$ of the patients with COVID-19, require intensive treatment such as ventilators and extracorporeal membrane oxygenation.

As the number of patients with COVID-19 increased especially in Europe and United States, the number of health care workers infected with COVID-19 also increased, placing aspects of the health care system such as emergency medicine and intensive care into a worldwide state of crisis.[3] The health care system in Japan is funded by public health insurance, and the emergency medical service (EMS) system, which handles all ambulance calls, is a free public service.[4] However, the impact of the COVID-19 pandemic on the EMS system has not been fully revealed, and little is known about the pandemic's impact on the incident number of emergency patients transported by ambulance and the number of deaths among emergency patients admitted to hospital. 
Osaka Prefecture is the largest metropolitan area in western Japan, with a population of 8.8 million. The annual number of ambulance calls is about 500,000 in this area, and that of patients transported to hospital by ambulance is about 200,000.[5] After the first patient in Osaka Prefecture was confirmed to have COVID-19 on 23 January 2020, the cumulative number of patients with COVID-19 in the prefecture rose to 1732 by 31 May, which was considered the first surge of COVID-19.[6] At that time, we revealed the influence of the COVID-19 pandemic on the EMS system in Osaka city.[7] The purpose of the present study was to determine the impact of the COVID-19 pandemic on the incident number of emergency patients transported by ambulance and the number of deaths among emergency patients admitted to hospital.

\section{Materials And Methods}

\subsection{Study design and settings}

This was a retrospective descriptive study with a study period from 1 January 2020 to 31 May 2020. We included patients transported by ambulance whose cleaned data was recorded in the ORION (Osaka emergency information Research Intelligent Operation Network) system. Therefore, we excluded patients who were not registered in the ORION system or who had missing data.

In $2019,8,823,452$ people lived in the $1905-\mathrm{km}^{2}$ area of Osaka Prefecture.[8] Of that population, 4,235,996 people (48.0\%) were male, and 2,382,016 people (27.0\%) were elderly, aged 65 years old or more. ORION data are considered administrative records and the ORION data is anonymized without specific personal data, such as patient name, date of birth, and address.

Therefore, the requirement of obtaining patient informed consent was waived.

This study was approved by the Ethics Committee of Osaka University Graduate School of Medicine (approval no.

15003). This manuscript was written based on the STROBE statement to assess the reporting of cohort and cross-sectional studies.[9] All methods in this study have ben carried out in accordance with the declaration of Helsinki.

\subsection{COVID-19 pandemic in Osaka Prefecture}

We previously revealed the characteristics and outcome of patients with COVID-19 in Osaka Prefecture.[6] Those patients in Osaka Prefecture suspected of having COVID-19 based on their medical and travel history were transferred to a hospital that specializes in the management of COVID-19 for PCR testing. When a COVID-19 outbreak was reported in places such as bars and live music venues, the staff in each public health centre in charge followed up on the people involved, and data on the individuals with positive PCR test results were collected to determine whether they were asymptomatic. All patients with positive PCR test results for COVID-19 were reported to the public health centres in accordance with the Infectious Disease Control Law.[10] In Osaka Prefecture, the first patient with COVID-19 was identified on 23 January, and by 31 May, 31,152 PCR tests had been conducted and the number of patients with 
COVID-19 was 1782. In Japan, due to an increase in the number of patients with COVID-19, the Japanese government declared a state of emergency based on the Law on 7 April 2020.

\subsection{EMS system and hospitals in Osaka Prefecture and Japan}

The EMS system is basically the same as that used in other areas of Japan, as previously described.[4] In Osaka Prefecture, EMS systems such as ambulance dispatch systems are operated by each local government, and ambulances are dispatched by calling 1-1-9. In 2019, the EMS system was operated by 27 fire departments (242 ambulances) and 24 fire control stations.[11] In 2016, there were 523 medical institutions (107,005 beds) in Osaka Prefecture,[12] of which 288 are emergency medical hospitals including 16 critical care centres that are designated to accept patients with life-threatening emergency diseases such as severe trauma and sepsis. Since the introduction of the ORION system, EMS personnel at the scene have selected the appropriate hospital for emergency patients rather than a dispatcher.

\subsection{ORION system}

Information on the system configuration of ORION was previously described in detail. $[5,13]$ The EMS personnel at the scene operate the ORION smartphone app for each emergency patient. All of the data input into the cell phone app, such as vital signs and time of the call to the hospital for acceptance, are also recorded. The cell phone app data are accumulated in the ORION cloud server, and in cooperation with the dispatched EMS personnel, data managers at each fire department directly input or upload the ambulance record of each emergency patient so that it can be connected with the app data. Furthermore, the operators of each hospital also directly input or upload the patient's hospital data, such as diagnoses and outcomes, after hospital acceptance. The results of aggregated data in the ORION system are fed back to every fire department and emergency hospital. The Department of Public Health of Osaka Prefecture can also analyse the effects of health policy on the emergency medical system using these collected data. The ORION system has been in place in all fire departments and emergency hospitals in Osaka Prefecture since January 2016.

\subsection{Data collection and quality control}

The ORION system checks for errors in the input in-hospital data, and the staff of each emergency hospital can correct them, if necessary. Through these tasks, cell phone app data, ambulance records, and the in-hospital data such as diagnosis and prognosis can be comprehensively registered for each patient transported by an ambulance. The registered data are cleaned by the Working Group to analyze the emergency medical care system in Osaka Prefecture. Among the collected and cleaned data, we excluded inconsistent data that did not contain all of the cell phone app data, ambulance records, and inhospital data such as diagnosis and prognosis. In addition, we also excluded patients whose sex as registered by the fire department did not match that registered by the hospital or whose sex identifier was missing. We also excluded patients whose age input by the fire department and that by the hospital differed by 3 years or more. When this difference was present, we defined the age input by the hospital as the patient's true age.[5]

\subsection{Endpoints}


The primary endpoints of this study were the incident number of patients transported by ambulance in each month of the study period, and the number of deaths among emergency patients admitted to hospital in each month.

\subsection{Statistical analysis}

First, we calculated the number of patients transported by ambulance by reason for ambulance call on a monthly basis from January to May 2020. As a control, we calculated the same data on a monthly basis from January to May 2019. Reason for ambulance call was divided into 'fire accident', 'natural disaster', 'water accident', 'traffic accident involving car, ship, or aircraft', 'injury, poisoning, and disease due to industrial accident', 'disease and injury due to sports', 'other injury', 'trauma due to assault', 'acute disease', 'interhospital transport', and 'others'. ${ }^{5,13}$ To evaluate the impact of the COVID-19 pandemic on the EMS system, we calculated the incidence rate of the number of emergency patients transported by ambulance. We also calculated the incidence rate ratio (IRR) and its 95\% confidence interval (Cl) using a Poisson regression model with the year 2019 as the reference. We categorized the patients by age group (children [0-19 years old], adult [20-64 years old], and elderly [65 years old and over]) and also calculated their respective IRR and $95 \% \mathrm{Cl}$ values. Next, we calculated the number of deaths among emergency patients admitted to hospital by reason for ambulance call in each month, and similarly calculated the IRR and its $95 \% \mathrm{Cl}$ values. The offset for calculating the IRR was set to the population of Osaka Prefecture in 2019 $\left(8,823,452\right.$ people) ${ }^{8}$ The death of emergency patients admitted to hospital was defined from the outcome at 21 days after hospital admission. In addition, in a subgroup analysis, we selected the patients transported by ambulance whose reason for ambulance call was 'acute disease' and similarly calculated the IRR and $95 \% \mathrm{Cl}$ values. Statistical analyses were performed using STATA version $16.0 \mathrm{MP}$ software (StataCorp LP, College Station, TX, USA).

\section{Results}

Figure 1 shows the patient flow in this study. Among the 180,362 patients registered in the ORION registry from January to May 2020, 77,735 patients were hospitalised, and 5172 patients were dead at 21 days after hospital admission. In contrast, among the 205,195 patients registered in the ORION system from January to May 2019, 83,851 patients were hospitalised, and 5237 patients were dead at 21 days after hospital admission.

Table 1 shows the number of emergency patients transported by ambulance and the IRR $(95 \% \mathrm{Cl})$ for each month during study period. The number of emergency patients transported by ambulance from January to May $2020(n=180,362)$ was significantly decreased from that transported from January to May 2019 ( $n=205,195)$ (IRR: $0.88,95 \% \mathrm{Cl}$ : 0.87-0.88). The most common reason for an ambulance call was 'acute disease' for 34,230 patients in 2019 and 30,849 patients in 2020. During the study period, the reasons for an ambulance call for which the number of emergency patients transported by ambulance decreased were 'traffic accident involving car, ship, or aircraft' (IRR: $0.82,95 \% \mathrm{Cl}: 0.80-0.84$ ), 'injury, poisoning, and disease due to industrial accident' (IRR: $0.79,95 \% \mathrm{Cl}: 0.73-0.85)$, 'disease and injury due to sport' (IRR: 0.37, 95\% Cl: 0.33-0.42), 'other injury' (IRR: 0.92, 95\% Cl: 0.90-0.93), 'trauma due to 
assault' (IRR: 0.91, 95\% Cl: 0.84-0.99), 'acute disease' (IRR: 0.88, 95\% Cl: 0.87-0.89), and 'interhospital transport' (IRR: $0.87,95 \% \mathrm{Cl}: 0.85-0.90)$. By month, the greatest decrease in the number of emergency patients transported by ambulance was in April (IRR: $0.77,95 \% \mathrm{Cl}: 0.76-0.78$ ), followed by May (IRR: $0.78,95 \% \mathrm{Cl}: 0.77-0.79)$. In the subgroup analysis by age group, the number of emergency patients transported by ambulance decreased among children during study period (IRR: $0.88,95 \% \mathrm{Cl}: 0.87-0.88$ ). However, for adults and the elderly, the number of emergency patients transported by ambulance decreased after March 2020 compared to that in 2019. 
Table 1

The number of emergency patients registered in the Osaka Emergency Information Research Intelligent Operation Network System

\begin{tabular}{|c|c|c|c|c|c|c|c|}
\hline \multicolumn{2}{|l|}{ Reason for ambulance call } & \multirow{2}{*}{$\begin{array}{l}\text { January } \\
61\end{array}$} & \multirow{2}{*}{$\begin{array}{l}\text { February } \\
38\end{array}$} & \multirow{2}{*}{$\begin{array}{l}\text { March } \\
43\end{array}$} & \multirow{2}{*}{$\begin{array}{l}\text { April } \\
37\end{array}$} & \multirow{2}{*}{$\begin{array}{l}\text { May } \\
37\end{array}$} & \multirow{2}{*}{$\begin{array}{l}\text { Total } \\
216\end{array}$} \\
\hline Fire accident & 2019 & & & & & & \\
\hline & 2020 & 59 & 45 & 31 & 23 & 33 & 191 \\
\hline & IRR & 0.97 & 1.18 & 0.72 & 0.62 & 0.89 & 0.88 \\
\hline & $(95 \% \mathrm{Cl})$ & $\begin{array}{l}(0.66- \\
1.41)\end{array}$ & $\begin{array}{l}(0.75- \\
1.87)\end{array}$ & $\begin{array}{l}(0.44- \\
1.17)\end{array}$ & $\begin{array}{l}(0.35- \\
1.07)\end{array}$ & $\begin{array}{l}(0.54- \\
1.47)\end{array}$ & $\begin{array}{l}(0.72- \\
1.08)\end{array}$ \\
\hline \multirow[t]{4}{*}{ Natural disaster } & 2019 & 0 & 0 & 0 & 0 & 0 & 0 \\
\hline & 2020 & 8 & 0 & 0 & 0 & 0 & 8 \\
\hline & IRR & NA & NA & NA & NA & NA & NA \\
\hline & $(95 \% \mathrm{Cl})$ & NA & NA & NA & NA & NA & NA \\
\hline \multirow[t]{4}{*}{ Water accident } & 2019 & 5 & 3 & 6 & 2 & 2 & 18 \\
\hline & 2020 & 3 & 4 & 2 & 6 & 3 & 18 \\
\hline & IRR & 0.60 & 1.33 & 0.33 & 3.00 & 1.50 & 1.00 \\
\hline & $(95 \% \mathrm{Cl})$ & $\begin{array}{l}(0.09- \\
3.08)\end{array}$ & $\begin{array}{l}(0.23- \\
9.10)\end{array}$ & $\begin{array}{l}(0.03- \\
1.86)\end{array}$ & $\begin{array}{l}(0.54- \\
30.39)\end{array}$ & $\begin{array}{l}(0.17- \\
17.96)\end{array}$ & $\begin{array}{l}(0.49- \\
2.04)\end{array}$ \\
\hline \multirow{4}{*}{$\begin{array}{l}\text { Traffic accident } \\
\text { involving car, ship, or } \\
\text { aircraft }\end{array}$} & 2019 & 2,620 & 2,510 & 2,997 & 3,248 & 3,026 & 14,401 \\
\hline & 2020 & 2,632 & 2,574 & 2,672 & 1,887 & 2,097 & 11,862 \\
\hline & IRR & 1.00 & 1.03 & 0.89 & 0.58 & 0.69 & 0.82 \\
\hline & $(95 \% \mathrm{Cl})$ & $\begin{array}{l}(0.95- \\
1.06)\end{array}$ & $\begin{array}{l}(0.97- \\
1.08)\end{array}$ & $\begin{array}{l}(0.85- \\
0.94)\end{array}$ & $\begin{array}{l}(0.55- \\
0.62)\end{array}$ & $\begin{array}{l}(0.66- \\
0.73)\end{array}$ & $\begin{array}{l}(0.80- \\
0.84)\end{array}$ \\
\hline \multirow{4}{*}{$\begin{array}{l}\text { Injury, poisoning, and } \\
\text { disease due to industrial } \\
\text { accident }\end{array}$} & 2019 & 348 & 321 & 370 & 365 & 374 & 1778 \\
\hline & 2020 & 279 & 317 & 274 & 282 & 250 & 1402 \\
\hline & IRR & 0.80 & 0.99 & 0.74 & 0.77 & 0.67 & 0.79 \\
\hline & $(95 \% \mathrm{Cl})$ & $\begin{array}{l}(0.68- \\
0.94)\end{array}$ & $\begin{array}{l}(0.84- \\
1.16)\end{array}$ & $\begin{array}{l}(0.63- \\
0.87)\end{array}$ & $\begin{array}{l}(0.66- \\
0.90)\end{array}$ & $\begin{array}{l}(0.57- \\
0.79)\end{array}$ & $\begin{array}{l}(0.73- \\
0.85)\end{array}$ \\
\hline \multirow{4}{*}{$\begin{array}{l}\text { Disease and injury due } \\
\text { to sport }\end{array}$} & 2019 & 135 & 166 & 232 & 232 & 252 & 1017 \\
\hline & 2020 & 142 & 144 & 50 & 23 & 16 & 375 \\
\hline & IRR & 1.05 & 0.87 & 0.22 & 0.10 & 0.06 & 0.37 \\
\hline & $(95 \% \mathrm{Cl})$ & $\begin{array}{l}(0.82- \\
1.34)\end{array}$ & $\begin{array}{l}(0.69- \\
1.09)\end{array}$ & $\begin{array}{l}(0.16- \\
0.29)\end{array}$ & $\begin{array}{l}(0.06- \\
0.15)\end{array}$ & $\begin{array}{l}(0.04- \\
0.11)\end{array}$ & $\begin{array}{l}(0.33- \\
0.42)\end{array}$ \\
\hline
\end{tabular}




\begin{tabular}{|c|c|c|c|c|c|c|c|}
\hline \multicolumn{2}{|c|}{ Reason for ambulance call } & \multirow{2}{*}{$\begin{array}{l}\text { January } \\
7,116\end{array}$} & \multirow{2}{*}{$\begin{array}{l}\text { February } \\
5,753\end{array}$} & \multirow{2}{*}{$\begin{array}{l}\text { March } \\
6,317\end{array}$} & \multirow{2}{*}{$\begin{array}{l}\text { April } \\
6,401\end{array}$} & \multirow{2}{*}{$\begin{array}{l}\text { May } \\
6,157\end{array}$} & \multirow{2}{*}{$\begin{array}{l}\text { Total } \\
31,744\end{array}$} \\
\hline Other injury & 2019 & & & & & & \\
\hline & 2020 & 6,931 & 6,140 & 5,911 & 5,001 & 5,169 & 29,152 \\
\hline & IRR & 0.97 & 1.07 & 0.94 & 0.78 & 0.84 & 0.92 \\
\hline & $(95 \% \mathrm{Cl})$ & $\begin{array}{l}(0.94- \\
1.01)\end{array}$ & $\begin{array}{l}(1.03- \\
1.11)\end{array}$ & $\begin{array}{l}(0.90- \\
0.97)\end{array}$ & $\begin{array}{l}(0.75- \\
0.81)\end{array}$ & $\begin{array}{l}(0.81- \\
0.87)\end{array}$ & $\begin{array}{l}(0.90- \\
0.93)\end{array}$ \\
\hline \multirow[t]{4}{*}{ Trauma due to assault } & 2019 & 268 & 207 & 232 & 232 & 224 & 1163 \\
\hline & 2020 & 251 & 221 & 228 & 170 & 190 & 1060 \\
\hline & IRR & 0.94 & 1.07 & 0.98 & 0.73 & 0.85 & 0.91 \\
\hline & $(95 \% \mathrm{Cl})$ & $\begin{array}{l}(0.79- \\
1.12)\end{array}$ & $\begin{array}{l}(0.88- \\
1.30)\end{array}$ & $\begin{array}{l}(0.81- \\
1.18)\end{array}$ & $\begin{array}{l}(0.60- \\
0.90)\end{array}$ & $\begin{array}{l}(0.70- \\
1.03)\end{array}$ & $\begin{array}{l}(0.84- \\
0.99)\end{array}$ \\
\hline \multirow[t]{4}{*}{ Self-induced injury } & 2019 & 197 & 195 & 245 & 216 & 254 & 1107 \\
\hline & 2020 & 265 & 217 & 250 & 183 & 250 & 1165 \\
\hline & IRR & 1.35 & 1.11 & 1.02 & 0.85 & 0.98 & 1.05 \\
\hline & $(95 \% \mathrm{Cl})$ & $\begin{array}{l}(1.11- \\
1.63)\end{array}$ & $\begin{array}{l}(0.91- \\
1.36)\end{array}$ & $\begin{array}{l}(0.85- \\
1.22)\end{array}$ & $\begin{array}{l}(0.69- \\
1.04)\end{array}$ & $\begin{array}{l}(0.82- \\
1.18)\end{array}$ & $\begin{array}{l}(0.97- \\
1.14)\end{array}$ \\
\hline \multirow[t]{4}{*}{ Acute disease } & 2019 & 34,240 & 25,757 & 26,545 & 26,371 & 27,525 & 140,438 \\
\hline & 2020 & 30,849 & 25,615 & 24,168 & 21,291 & 21,587 & 123,510 \\
\hline & IRR & 0.90 & 0.99 & 0.91 & 0.81 & 0.78 & 0.88 \\
\hline & $(95 \% \mathrm{Cl})$ & $\begin{array}{l}(0.89- \\
0.91)\end{array}$ & $\begin{array}{l}(0.98- \\
1.01)\end{array}$ & $\begin{array}{l}(0.89- \\
0.93)\end{array}$ & $\begin{array}{l}(0.79- \\
0.82)\end{array}$ & $\begin{array}{l}(0.77- \\
0.80)\end{array}$ & $\begin{array}{l}(0.87- \\
0.89)\end{array}$ \\
\hline \multirow[t]{4}{*}{ Interhospital transport } & 2019 & 2,897 & 2,445 & 2,626 & 2,732 & 2,553 & 13,253 \\
\hline & 2020 & 2,895 & 2,449 & 2,360 & 1,918 & 1,951 & 11,573 \\
\hline & IRR & 1.00 & 1.00 & 0.90 & 0.70 & 0.76 & 0.87 \\
\hline & $(95 \% \mathrm{Cl})$ & $\begin{array}{l}(0.95- \\
1.05)\end{array}$ & $\begin{array}{l}(0.95- \\
1.06)\end{array}$ & $\begin{array}{l}(0.85- \\
0.95)\end{array}$ & $\begin{array}{l}(0.66- \\
0.74)\end{array}$ & $\begin{array}{l}(0.72- \\
0.81)\end{array}$ & $\begin{array}{l}(0.85- \\
0.90)\end{array}$ \\
\hline \multirow[t]{4}{*}{ Other } & 2019 & 14 & 9 & 13 & 11 & 13 & 60 \\
\hline & 2020 & 9 & 7 & 8 & 11 & 11 & 46 \\
\hline & IRR & 0.64 & 0.78 & 0.62 & 1.00 & 0.85 & 0.77 \\
\hline & $(95 \% \mathrm{Cl})$ & $\begin{array}{l}(0.25- \\
1.59)\end{array}$ & $\begin{array}{l}(0.25- \\
0.35)\end{array}$ & $\begin{array}{l}(0.22- \\
1.60)\end{array}$ & $\begin{array}{l}(0.39- \\
2.54)\end{array}$ & $\begin{array}{l}(0.34- \\
2.05)\end{array}$ & $\begin{array}{l}(0.51- \\
1.14)\end{array}$ \\
\hline Total & 2019 & 47,901 & 37,404 & 39,626 & 39,847 & 40,417 & 205,195 \\
\hline
\end{tabular}




\begin{tabular}{|c|c|c|c|c|c|c|c|}
\hline \multicolumn{2}{|c|}{ Reason for ambulance call } & \multirow{2}{*}{$\begin{array}{l}\text { January } \\
44,323\end{array}$} & \multirow{2}{*}{$\begin{array}{l}\text { February } \\
37,733\end{array}$} & \multirow{2}{*}{$\begin{array}{l}\text { March } \\
35,954\end{array}$} & \multirow{2}{*}{$\begin{array}{l}\text { April } \\
30,795\end{array}$} & \multirow{2}{*}{$\begin{array}{l}\text { May } \\
31,557\end{array}$} & \multirow{2}{*}{$\begin{array}{l}\text { Total } \\
180,362\end{array}$} \\
\hline & 2020 & & & & & & \\
\hline & IRR & 0.93 & 1.01 & 0.91 & 0.77 & 0.78 & 0.88 \\
\hline & $(95 \% \mathrm{Cl})$ & $\begin{array}{l}(0.91- \\
0.94)\end{array}$ & $\begin{array}{l}(0.99- \\
1.02)\end{array}$ & $\begin{array}{l}(0.89- \\
0.92)\end{array}$ & $\begin{array}{l}(0.76- \\
0.78)\end{array}$ & $\begin{array}{l}(0.77- \\
0.79)\end{array}$ & $\begin{array}{l}(0.87- \\
0.88)\end{array}$ \\
\hline \multirow{4}{*}{$\begin{array}{l}\text { Children aged 0-19 } \\
\text { years old }\end{array}$} & 2019 & 5,108 & 3,603 & 3,937 & 4,407 & 4,565 & 21,620 \\
\hline & 2020 & 4,198 & 3,211 & 2,762 & 2,262 & 2,287 & 14,720 \\
\hline & IRR & 0.82 & 0.89 & 0.70 & 0.51 & 0.50 & 0.68 \\
\hline & $(95 \% \mathrm{Cl})$ & $\begin{array}{l}(0.79- \\
0.86)\end{array}$ & $\begin{array}{l}(0.85- \\
0.93)\end{array}$ & $\begin{array}{l}(0.67- \\
0.74)\end{array}$ & $\begin{array}{l}(0.49- \\
0.54)\end{array}$ & $\begin{array}{l}(0.48- \\
0.53)\end{array}$ & $\begin{array}{l}(0.67- \\
0.70)\end{array}$ \\
\hline \multirow{4}{*}{$\begin{array}{l}\text { Adults aged 20-64 } \\
\text { years old }\end{array}$} & 2019 & 13,926 & 11,520 & 12,826 & 12,783 & 13,120 & 64,175 \\
\hline & 2020 & 13,442 & 11,617 & 11,614 & 10,001 & 10,402 & 57,076 \\
\hline & IRR & 0.97 & 1.01 & 0.91 & 0.78 & 0.79 & 0.89 \\
\hline & $(95 \% \mathrm{Cl})$ & $\begin{array}{l}(0.94- \\
0.99)\end{array}$ & $\begin{array}{l}(0.98- \\
1.03)\end{array}$ & $\begin{array}{l}(0.88- \\
0.93)\end{array}$ & $\begin{array}{l}(0.76- \\
0.80)\end{array}$ & $\begin{array}{l}(0.77- \\
0.81)\end{array}$ & $\begin{array}{l}(0.88- \\
0.90)\end{array}$ \\
\hline \multirow{4}{*}{$\begin{array}{l}\text { Elderlies aged }>=65 \\
\text { years old }\end{array}$} & 2019 & 28,867 & 22,281 & 22,863 & 22,657 & 22,732 & 119,400 \\
\hline & 2020 & 26,683 & 22,905 & 21,578 & 18,532 & 18,868 & 108,566 \\
\hline & IRR & 0.92 & 1.03 & 0.94 & 0.82 & 0.83 & 0.91 \\
\hline & $(95 \% \mathrm{Cl})$ & $\begin{array}{l}(0.91- \\
0.94)\end{array}$ & $\begin{array}{l}(1.01- \\
1.05)\end{array}$ & $\begin{array}{l}(0.93- \\
0.96)\end{array}$ & $\begin{array}{l}(0.80- \\
0.83)\end{array}$ & $\begin{array}{l}(0.81- \\
0.85)\end{array}$ & $\begin{array}{l}(0.90- \\
0.92)\end{array}$ \\
\hline \multicolumn{8}{|c|}{ IRR: incident rate ratio, Cl: confidence interval, NA: not assessment. } \\
\hline \multicolumn{8}{|c|}{ IRR is for 2020 versus 2019.} \\
\hline
\end{tabular}

Table 2 shows the number of deaths among emergency patients admitted to hospital and the IRR (95\% $\mathrm{Cl}$ ) for each month. The number of deaths among emergency patients admitted to hospital was 5237 in January-May 2019 and remained essentially unchanged at 5172 in January-May 2020 (IRR: 0.99, 95\% Cl: 0.95-1.03). There was no statistically significant change in the number of deaths among emergency patients admitted to hospital for each reason for an ambulance call between 2019 and 2020, and no statistically significant differences were identified between 2019 and 2020 for each month. In subgroup analysis by age group, there was no increase of the number of deaths of emergency patients admitted to hospital among children (IRR: $0.84,95 \% \mathrm{Cl}$ : 0.45-1.09), adults (IRR: $0.93,95 \% \mathrm{Cl}$ : $0.83-1.04$ ), and the elderly (IRR: 1.00, 95\% Cl: 0.96-1.04). 
Table 2

The number of death among hospitalized emergency patients registered in the ORION system

\begin{tabular}{|c|c|c|c|c|c|c|c|}
\hline \multicolumn{2}{|l|}{ Reason for ambulance call } & \multirow{2}{*}{$\begin{array}{l}\text { January } \\
3\end{array}$} & \multirow{2}{*}{$\begin{array}{l}\text { February } \\
1\end{array}$} & \multirow{2}{*}{$\begin{array}{l}\text { March } \\
0\end{array}$} & \multirow{2}{*}{$\begin{array}{l}\text { April } \\
2\end{array}$} & \multirow{2}{*}{$\begin{array}{l}\text { May } \\
2\end{array}$} & \multirow{2}{*}{$\begin{array}{l}\text { Total } \\
8\end{array}$} \\
\hline Fire accident & 2019 & & & & & & \\
\hline & 2020 & 4 & 3 & 1 & 1 & 1 & 10 \\
\hline & IRR & 1.33 & 3.00 & NA & 0.50 & 0.50 & 1.25 \\
\hline & $(95 \% \mathrm{Cl})$ & $\begin{array}{l}(0.23- \\
0.910)\end{array}$ & $\begin{array}{l}(0.24- \\
157.49)\end{array}$ & NA & $\begin{array}{l}(0.01- \\
9.60)\end{array}$ & $\begin{array}{l}(0.01- \\
9.60)\end{array}$ & $\begin{array}{l}(0.44- \\
3.64)\end{array}$ \\
\hline \multirow[t]{4}{*}{ Natural disaster } & 2019 & 0 & 0 & 0 & 0 & 0 & 0 \\
\hline & 2020 & 0 & 0 & 0 & 0 & 0 & 0 \\
\hline & IRR & NA & NA & NA & NA & NA & NA \\
\hline & $(95 \% \mathrm{Cl})$ & NA & NA & NA & NA & NA & NA \\
\hline \multirow[t]{4}{*}{ Water accident } & 2019 & 0 & 0 & 0 & 0 & 1 & 1 \\
\hline & 2020 & 0 & 0 & 0 & 1 & 0 & 1 \\
\hline & IRR & NA & NA & NA & NA & NA & 1.00 \\
\hline & $(95 \% \mathrm{Cl})$ & NA & NA & NA & NA & NA & $\begin{array}{l}(0.01- \\
78.50)\end{array}$ \\
\hline \multirow{4}{*}{$\begin{array}{l}\text { Traffic accident involving } \\
\text { car, ship, or aircraft }\end{array}$} & 2019 & 8 & 7 & 9 & 11 & 7 & 42 \\
\hline & 2020 & 8 & 8 & 13 & 6 & 7 & 42 \\
\hline & IRR & 1.00 & 1.14 & 1.44 & 0.55 & 1.00 & 1.00 \\
\hline & $(95 \% \mathrm{Cl})$ & $\begin{array}{l}(0.33- \\
3.06)\end{array}$ & $\begin{array}{l}(0.36- \\
3.70)\end{array}$ & $\begin{array}{l}(0.57- \\
3.83)\end{array}$ & $\begin{array}{l}(0.17- \\
1.61)\end{array}$ & $\begin{array}{l}(0.30- \\
3.34)\end{array}$ & $\begin{array}{l}(0.64- \\
1.57)\end{array}$ \\
\hline \multirow{4}{*}{$\begin{array}{l}\text { Injury, poisoning, and } \\
\text { disease due to industrial } \\
\text { accident }\end{array}$} & 2019 & 2 & 0 & 1 & 0 & 3 & 6 \\
\hline & 2020 & 3 & 1 & 0 & 4 & 0 & 8 \\
\hline & IRR & 1.50 & NA & NA & NA & NA & 1.33 \\
\hline & $(95 \% \mathrm{Cl})$ & $\begin{array}{l}(0.17- \\
17.96)\end{array}$ & NA & NA & NA & NA & $\begin{array}{l}(0.41- \\
4.66)\end{array}$ \\
\hline \multirow{4}{*}{$\begin{array}{l}\text { Disease and injury due } \\
\text { to sport }\end{array}$} & 2019 & 0 & 1 & 0 & 0 & 0 & 1 \\
\hline & 2020 & 1 & 0 & 0 & 0 & 0 & 1 \\
\hline & IRR & NA & NA & NA & NA & NA & 1.00 \\
\hline & $(95 \% \mathrm{Cl})$ & NA & NA & NA & NA & NA & $\begin{array}{l}(0.01- \\
78.50)\end{array}$ \\
\hline
\end{tabular}




\begin{tabular}{|c|c|c|c|c|c|c|c|}
\hline \multicolumn{2}{|c|}{ Reason for ambulance call } & \multirow{2}{*}{$\begin{array}{l}\text { January } \\
73\end{array}$} & \multirow{2}{*}{$\begin{array}{l}\text { February } \\
57\end{array}$} & \multirow{2}{*}{$\begin{array}{l}\text { March } \\
33\end{array}$} & \multirow{2}{*}{$\begin{array}{l}\text { April } \\
50\end{array}$} & \multirow{2}{*}{$\begin{array}{l}\text { May } \\
36\end{array}$} & \multirow{2}{*}{$\begin{array}{l}\text { Total } \\
249\end{array}$} \\
\hline Other injury & 2019 & & & & & & \\
\hline & 2020 & 61 & 41 & 47 & 36 & 35 & 220 \\
\hline & IRR & 0.84 & 0.72 & 1.42 & 0.72 & 0.97 & 0.88 \\
\hline & $(95 \% \mathrm{Cl})$ & $\begin{array}{l}(0.58- \\
1.19)\end{array}$ & $\begin{array}{l}(0.47- \\
1.09)\end{array}$ & $\begin{array}{l}(0.89- \\
2.29)\end{array}$ & $\begin{array}{l}(0.46- \\
1.13)\end{array}$ & $\begin{array}{l}(0.59- \\
1.59)\end{array}$ & $\begin{array}{l}(0.73- \\
1.06)\end{array}$ \\
\hline \multirow[t]{4}{*}{ Trauma due to assault } & 2019 & 0 & 0 & 0 & 2 & 0 & 2 \\
\hline & 2020 & 0 & 1 & 0 & 0 & 0 & 1 \\
\hline & IRR & NA & NA & NA & NA & NA & 0.50 \\
\hline & $(95 \% \mathrm{Cl})$ & NA & NA & NA & NA & NA & $\begin{array}{l}(0.01- \\
9.60)\end{array}$ \\
\hline \multirow[t]{4}{*}{ Self-induced injury } & 2019 & 8 & 6 & 7 & 15 & 13 & 49 \\
\hline & 2020 & 8 & 10 & 11 & 8 & 11 & 48 \\
\hline & IRR & 1.00 & 1.67 & 1.57 & 0.53 & 0.85 & 0.98 \\
\hline & $(95 \% \mathrm{Cl})$ & $\begin{array}{l}(0.33- \\
3.06)\end{array}$ & $\begin{array}{l}(0.55- \\
5.58)\end{array}$ & $\begin{array}{l}(0.56- \\
4.78)\end{array}$ & $\begin{array}{l}(0.20- \\
1.34)\end{array}$ & $\begin{array}{l}(0.34- \\
2.05)\end{array}$ & $\begin{array}{l}(0.64- \\
1.49)\end{array}$ \\
\hline \multirow[t]{4}{*}{ Acute disease } & 2019 & 1,112 & 829 & 870 & 770 & 767 & 4,348 \\
\hline & 2020 & 1,028 & 911 & 880 & 753 & 742 & 4,314 \\
\hline & IRR & 0.92 & 1.10 & 1.01 & 0.98 & 0.97 & 0.99 \\
\hline & $(95 \% \mathrm{Cl})$ & $\begin{array}{l}(0.85- \\
1.01)\end{array}$ & $\begin{array}{l}(1.00- \\
1.21)\end{array}$ & $\begin{array}{l}(0.92- \\
1.11)\end{array}$ & $\begin{array}{l}(0.88- \\
1.08)\end{array}$ & $\begin{array}{l}(0.87- \\
1.07)\end{array}$ & $\begin{array}{l}(0.95- \\
1.04)\end{array}$ \\
\hline \multirow[t]{4}{*}{ Interhospital transport } & 2019 & 119 & 117 & 86 & 110 & 98 & 530 \\
\hline & 2020 & 138 & 92 & 104 & 97 & 93 & 524 \\
\hline & IRR & 1.16 & 0.79 & 1.21 & 0.88 & 0.95 & 0.99 \\
\hline & $(95 \% \mathrm{Cl})$ & $\begin{array}{l}(0.90- \\
1.49)\end{array}$ & $\begin{array}{l}(0.59- \\
1.04)\end{array}$ & $\begin{array}{l}(0.90- \\
1.63)\end{array}$ & $\begin{array}{l}(0.66- \\
1.17)\end{array}$ & $\begin{array}{l}(0.71- \\
1.27)\end{array}$ & $\begin{array}{l}(0.87- \\
1.12)\end{array}$ \\
\hline \multirow[t]{4}{*}{ Other } & 2019 & 0 & 0 & 0 & 1 & 0 & 1 \\
\hline & 2020 & 0 & 1 & 0 & 0 & 2 & 3 \\
\hline & IRR & NA & NA & NA & NA & NA & 3.00 \\
\hline & $(95 \% \mathrm{Cl})$ & NA & NA & NA & NA & NA & $\begin{array}{l}(0.24- \\
157.49)\end{array}$ \\
\hline Total & 2019 & 1,325 & 1,018 & 1,006 & 961 & 927 & 5,237 \\
\hline
\end{tabular}




\begin{tabular}{|c|c|c|c|c|c|c|c|}
\hline \multicolumn{2}{|l|}{ Reason for ambulance call } & \multirow{2}{*}{$\begin{array}{l}\text { January } \\
1,251\end{array}$} & \multirow{2}{*}{$\begin{array}{l}\text { February } \\
1,068\end{array}$} & \multirow{2}{*}{$\begin{array}{l}\text { March } \\
1,056\end{array}$} & \multirow{2}{*}{$\begin{array}{l}\text { April } \\
906\end{array}$} & \multirow{2}{*}{$\begin{array}{l}\text { May } \\
891\end{array}$} & \multirow{2}{*}{$\begin{array}{l}\text { Total } \\
5,172\end{array}$} \\
\hline & 2020 & & & & & & \\
\hline & $\begin{array}{l}\text { IRR } \\
(95 \% \\
\text { Cl) }\end{array}$ & 0.94 & 1.05 & 1.05 & 0.94 & 0.96 & 0.99 \\
\hline & $(95 \% \mathrm{Cl})$ & $\begin{array}{l}(0.87- \\
1.02)\end{array}$ & $\begin{array}{l}(0.96- \\
1.14)\end{array}$ & $\begin{array}{l}(0.96- \\
1.15)\end{array}$ & $\begin{array}{l}(0.86- \\
1.03)\end{array}$ & $\begin{array}{l}(0.88- \\
1.05)\end{array}$ & $\begin{array}{l}(0.95- \\
1.03)\end{array}$ \\
\hline \multirow{4}{*}{$\begin{array}{l}\text { Children aged 0-19 } \\
\text { years old }\end{array}$} & 2019 & 9 & 2 & 4 & 7 & 3 & 25 \\
\hline & 2020 & 5 & 8 & 4 & 3 & 1 & 21 \\
\hline & $\begin{array}{l}\text { IRR } \\
(95 \% \\
\mathrm{Cl})\end{array}$ & 0.56 & 4.00 & 1.00 & 0.43 & 0.33 & 0.84 \\
\hline & $(95 \% \mathrm{Cl})$ & $\begin{array}{l}(0.15- \\
1.85)\end{array}$ & $\begin{array}{l}(0.80- \\
38.67)\end{array}$ & $\begin{array}{l}(0.19- \\
5.37)\end{array}$ & $\begin{array}{l}(0.07- \\
1.88)\end{array}$ & $\begin{array}{l}(0.01- \\
4.15)\end{array}$ & $\begin{array}{l}(0.45- \\
1.56)\end{array}$ \\
\hline \multirow{4}{*}{$\begin{array}{l}\text { Adults aged } 20-64 \text { years } \\
\text { old }\end{array}$} & 2019 & 173 & 115 & 123 & 122 & 110 & 643 \\
\hline & 2020 & 155 & 111 & 114 & 124 & 94 & 598 \\
\hline & $\begin{array}{l}\text { IRR } \\
(95 \% \\
\text { Cl) }\end{array}$ & 0.90 & 0.97 & 0.93 & 1.02 & 0.85 & 0.93 \\
\hline & $(95 \% \mathrm{Cl})$ & $\begin{array}{l}(0.72- \\
1.12)\end{array}$ & $\begin{array}{l}(0.74- \\
1.26)\end{array}$ & $\begin{array}{l}(0.71- \\
1.21)\end{array}$ & $\begin{array}{l}(0.79- \\
1.32)\end{array}$ & $\begin{array}{l}(0.64- \\
1.14)\end{array}$ & $\begin{array}{l}(0.83- \\
1.04)\end{array}$ \\
\hline \multirow{4}{*}{$\begin{array}{l}\text { Elderlies aged }>=65 \\
\text { years old }\end{array}$} & 2019 & 1,143 & 901 & 879 & 832 & 814 & 4,569 \\
\hline & 2020 & 1,091 & 949 & 938 & 779 & 796 & 4,553 \\
\hline & $\begin{array}{l}\text { IRR } \\
(95 \% \\
\text { Cl) }\end{array}$ & 0.95 & 1.05 & 1.07 & 0.94 & 0.98 & 1.00 \\
\hline & $(95 \% \mathrm{Cl})$ & $\begin{array}{l}(0.88- \\
1.04)\end{array}$ & $\begin{array}{l}(0.96- \\
1.16)\end{array}$ & $\begin{array}{l}(0.97- \\
1.17)\end{array}$ & $\begin{array}{l}(0.85- \\
1.03)\end{array}$ & $\begin{array}{l}(0.89- \\
1.08)\end{array}$ & $\begin{array}{l}(0.96- \\
1.04)\end{array}$ \\
\hline \multicolumn{8}{|c|}{ IRR: incident rate ratio, Cl: confidence interval, NA: not assessment. } \\
\hline \multicolumn{8}{|l|}{ IRR is for 2020 versus 2019.} \\
\hline
\end{tabular}

Table 3 shows the number of emergency patients transported by ambulance due to acute disease by age group and the IRR $(95 \% \mathrm{Cl})$ for each month during the study period. The number of paediatric patients transported by ambulance during the study period significantly decreased $(13,164$ patients in JanuaryMay 2019 vs 8483 patients in January-May 2020; IRR: 0.64, 95\% Cl: 0.63-0.66). The number of adult patients transported by ambulance also significantly decreased (42,746 patients in January-May 2019 vs 
$38,671$ patients in January-May 2020, IRR: $0.90,95 \% \mathrm{Cl}: 0.89-0.92)$, as did that of the elderly patients transported by ambulance (84,528 patients in January-May 2019 vs 76,356 patients in January-May 2020, IRR: 0.90, 95\% Cl: 0.89-0.91).

Table 3

The number of emergency patients for acute disease registered in the ORION system

\begin{tabular}{|c|c|c|c|c|c|c|c|}
\hline Acute disease & & January & February & March & April & May & Total \\
\hline \multirow{4}{*}{$\begin{array}{l}\text { Childen aged 0-19 } \\
\text { years old }\end{array}$} & 2019 & 3,629 & 2,273 & 2,219 & 2,451 & 2,592 & 13,164 \\
\hline & 2020 & 2,837 & 1,968 & 1,496 & 1,158 & 1,024 & 8,483 \\
\hline & IRR & 0.78 & 0.87 & 0.67 & 0.47 & 0.40 & 0.64 \\
\hline & $(95 \% \mathrm{Cl})$ & $\begin{array}{l}(0.74- \\
0.82)\end{array}$ & $\begin{array}{l}(0.81- \\
0.92)\end{array}$ & $\begin{array}{l}(0.63- \\
0.72)\end{array}$ & $\begin{array}{l}(0.44- \\
0.51)\end{array}$ & $\begin{array}{l}(0.37- \\
0.42)\end{array}$ & $\begin{array}{l}(0.63- \\
0.66)\end{array}$ \\
\hline \multirow{4}{*}{$\begin{array}{l}\text { Adults aged 20-64 } \\
\text { years old }\end{array}$} & 2019 & 9,748 & 7,644 & 8,369 & 8,266 & 8,719 & 42,746 \\
\hline & 2020 & 9,235 & 7,656 & 7,615 & 7,002 & 7,163 & 38,671 \\
\hline & IRR & 0.95 & 1.00 & 0.91 & 0.85 & 0.82 & 0.90 \\
\hline & $(95 \% \mathrm{Cl})$ & $\begin{array}{l}(0.92- \\
0.97)\end{array}$ & $\begin{array}{l}(0.97- \\
1.03)\end{array}$ & $\begin{array}{l}(0.88- \\
0.94)\end{array}$ & $\begin{array}{l}(0.82- \\
0.87)\end{array}$ & $\begin{array}{l}(0.80- \\
0.85)\end{array}$ & $\begin{array}{l}(0.89- \\
0.92)\end{array}$ \\
\hline \multirow{4}{*}{$\begin{array}{l}\text { Elderlies aged }>=65 \\
\text { years old }\end{array}$} & 2019 & 20,863 & 15,840 & 15,957 & 15,654 & 16,214 & 84,528 \\
\hline & 2020 & 18,777 & 15,991 & 15,057 & 13,131 & 13,400 & 76,356 \\
\hline & IRR & 0.90 & 1.01 & 0.94 & 0.84 & 0.83 & 0.90 \\
\hline & $(95 \% \mathrm{Cl})$ & $\begin{array}{l}(0.88- \\
0.92)\end{array}$ & $\begin{array}{l}(0.99- \\
1.03)\end{array}$ & $\begin{array}{l}(0.92- \\
0.96)\end{array}$ & $(0.82-.86)$ & $\begin{array}{l}(0.81- \\
0.85)\end{array}$ & $\begin{array}{l}(0.89- \\
0.91)\end{array}$ \\
\hline \multicolumn{8}{|c|}{ IRR: incident rate ratio, Cl: confidence interval. } \\
\hline \multicolumn{8}{|c|}{ IRR is for 2020 versus 2019.} \\
\hline
\end{tabular}

Table 4 shows the number of deaths among emergency patients admitted to hospital due to acute disease by age group and IRR $(95 \% \mathrm{Cl})$ for each month. The number of deaths among emergency paediatric patients admitted to hospital due to acute disease was 11 in January-May 2019 and 11 in January-May 2020 (IRR: 1.00, 95\% Cl: 0.39-2.54). The number of deaths among emergency adult patients admitted to hospital due to acute disease was 509 in January-May 2019 and 469 in JanuaryMay 2020 (IRR: $0.92,95 \% \mathrm{Cl}: 0.81-1.05$ ), and that among emergency elderly patients admitted to hospital due to acute disease was 3828 in January-May 2019 and 3834 in January-May 2020 (IRR: 1.00, $95 \% \mathrm{Cl}:$ 0.96-1.05). No statistically significant differences were identified between 2019 and 2020 for each month or by age group. 
Table 4

The number of death among hospitalized emergency patients for acute disease registered in the ORION system

\begin{tabular}{|c|c|c|c|c|c|c|c|}
\hline Acute disease & & January & February & March & April & May & Total \\
\hline \multirow{4}{*}{$\begin{array}{l}\text { Childen aged 0-19 } \\
\text { years old }\end{array}$} & 2019 & 4 & 2 & 1 & 2 & 2 & 11 \\
\hline & 2020 & 4 & 2 & 2 & 2 & 1 & 11 \\
\hline & IRR & 1.00 & 1.00 & 2.00 & 1.00 & 0.50 & 1.00 \\
\hline & $(95 \% \mathrm{Cl})$ & $\begin{array}{l}(0.19- \\
5.37)\end{array}$ & $\begin{array}{l}(0.07- \\
13.80)\end{array}$ & $\begin{array}{l}(0.10- \\
117.99)\end{array}$ & $\begin{array}{l}(0.07- \\
13.80)\end{array}$ & $\begin{array}{l}(0.01- \\
9.60)\end{array}$ & $\begin{array}{l}(0.39- \\
2.54)\end{array}$ \\
\hline \multirow{4}{*}{$\begin{array}{l}\text { Adults aged 20-64 } \\
\text { years old }\end{array}$} & 2019 & 143 & 84 & 107 & 96 & 79 & 509 \\
\hline & 2020 & 124 & 89 & 87 & 94 & 75 & 469 \\
\hline & IRR & 0.87 & 1.06 & 0.81 & 0.98 & 0.95 & 0.92 \\
\hline & $(95 \% \mathrm{Cl})$ & $\begin{array}{l}(0.68- \\
1.11)\end{array}$ & $\begin{array}{l}(0.78- \\
1.44)\end{array}$ & $\begin{array}{l}(0.61- \\
1.09)\end{array}$ & $\begin{array}{l}(0.73- \\
1.32)\end{array}$ & $\begin{array}{l}(0.68- \\
1.32)\end{array}$ & $\begin{array}{l}(0.81- \\
1.05)\end{array}$ \\
\hline \multirow{4}{*}{$\begin{array}{l}\text { Elderlies aged > = } 65 \\
\text { years old }\end{array}$} & 2019 & 965 & 743 & 762 & 672 & 686 & 3,828 \\
\hline & 2020 & 900 & 820 & 791 & 657 & 666 & 3,834 \\
\hline & IRR & 0.93 & 1.10 & 1.04 & 0.98 & 0.97 & 1.00 \\
\hline & $(95 \% \mathrm{Cl})$ & $\begin{array}{l}(0.85- \\
1.02)\end{array}$ & $\begin{array}{l}(1.00- \\
1.22)\end{array}$ & $\begin{array}{l}(0.94- \\
1.15)\end{array}$ & $\begin{array}{l}(0.88- \\
1.09)\end{array}$ & $\begin{array}{l}(0.87- \\
1.08)\end{array}$ & $\begin{array}{l}(0.96- \\
1.05)\end{array}$ \\
\hline \multicolumn{8}{|c|}{ IRR: incident rate ratio, $\mathrm{Cl}$ : confidence interval. } \\
\hline \multicolumn{8}{|c|}{ IRR is for 2020 versus 2019.} \\
\hline
\end{tabular}

\section{Discussion}

In this study, we used data from a large population-based patient registry to determine the number of emergency patients transported by ambulance and the number of deaths among these patients admitted to hospital in the first surge of the COVID-19 pandemic in Osaka Prefecture. Although the number of emergency patients transported by ambulance decreased in 2020 compared with 2019, the number of deaths among the emergency patients admitted to hospital in 2020 was similar to that in 2019 . The results of this study, which used population-based data to reveal the impact of an emerging infectious disease pandemic on the EMS system, could be useful to plan health care systems and policies.

The number of emergency patients transported by ambulance decreased in 2020 compared with 2019, especially from March to May. As well, the number of emergency patients transported by ambulance due to acute disease as the reason for the ambulance call also decreased, especially in April and May. A 
previous study in Venice, northern Italy, comparing the number of ambulance dispatches in 2019 and 2020 found that the COVID-19 pandemic reduced the number of ambulance dispatches in 2020.[14] It was also reported that the number of emergency department visits decreased during the severe acute respiratory syndrome (SARS) pandemic that spread in 2003.[15-19] Thus, when an infectious disease spreads throughout a city or society, the number of emergency department visits may decrease as a result of people buying medicines from pharmacies for their own care and refraining from visiting the emergency department. In contrast, in Seine-Saint-Denis, which is a French department bordering Paris to the northeast and is a part of Greater Paris, Lapostolle et al. reported that the COVID-19 pandemic increased the number of calls for the Service d'Aide Medicale Urgente (SAMU), the number of mobile intensive care unit (MICU) dispatches, and the number of emergency department visits compared to the average of the previous five years.[20] Although the reason for the differences between their study and the present study is not clear, the SAMU in France provides several medical services that included medical advice, hospital transfer by a non-emergency transport ambulance, dispatch of a rescue vehicle or of a general practitioner to the patient's home or, in severe cases and/or when rapid prehospital care is required, a MICU, with an emergency physician, nurse, and special paramedic on board. Contrastingly, the only service provided by the EMS system in Japan is ambulance dispatch, and the differences in services provided by the SAMU in France versus the EMS system in Japan may have affected the difference in results. Further, Saberian et al. reported an increase in the number of EMS calls and ambulance dispatches after the first COVID-19 patient was identified on 18 February 2020 in Teheran, Iran.[21] The EMS system in Iran is similar to that in Japan in that the EMS personnel evaluate the patient at the scene and, if necessary, transport the patient to a hospital. The difference of results between the study in Japan and that in Iran, which operates a similar EMS system, may be due to the fact that Japanese people who used to call an ambulance even in cases not necessarily requiring an ambulance are now discouraged from visiting hospitals and clinics due the risk of COVID-19.

The number of emergency patients transported by ambulance due to sports injuries, industrial accidents, and traffic accidents also decreased in 2020 compared to 2019. In Japan, the Japanese government requested temporary closures of elementary, junior high, and high schools on 2 March,[22] and the temporary closure of these schools continued until 31 May 2020 in Osaka Prefecture. In addition, many sports gyms have refrained from operating as a result of COVID-19 outbreaks in some of these gyms. As a result of this reduction in opportunities for sports in schools and gyms, the number of emergency patients transported by ambulance due to sports injuries would likely have decreased. In Japan, although no explicit lockdown measures were taken by the government, the number of emergency patients transported by ambulance due to traffic accidents and industrial accidents may have also decreased because of the slowdown in socioeconomic activity due to the voluntary restraint of various companies. Subgroup analyses by age group showed a decrease in patients transported by ambulance among children starting in January and a decrease in patients transported by ambulance among adults and elderly after March. This result may be due to parents being less likely to visit the emergency department due to vigilance against an unknown infectious disease. In addition, as a result of school closures, they 
may not have visited emergency departments as a result of fewer cases of seasonal influenza in their children.

There was no change in the number of deaths among emergency patients admitted to hospital in 2020 compared with 2019. There were also no differences in the number of deaths among emergency patients admitted to hospital in the analyses by reason for ambulance call or by age group. Additionally, there were concerns that other acute illnesses might affect the prognosis of emergency elderly patients. However, no impact on their prognosis was identified in this study because the health care system and EMS system functioned effectively for the community as a whole. To maintain the level of medical treatment in future surges of the COVID-19 pandemic and other infectious disease pandemics, it will be necessary to establish a medical and health care system with a clear role for medical institutions.

This study has several limitations. First, although all fire departments and emergency medical institutions in Osaka Prefecture registered ambulance records and patient data in the ORION registry, the prognosis of patients transported to medical institutions outside Osaka Prefecture or by fire departments outside Osaka Prefecture is unknown. Second, no information was available on the detailed treatment of the patients in hospital that would have affected death after hospital admission. Third, although this study was analysed by reason for ambulance call, a detailed analysis of the impact of the COVID-19 pandemic on the EMS system by disease, such as out-of-hospital cardiac arrest, acute coronary syndrome, and pneumonia, will be performed and reported in the near future.

In conclusion, in Osaka Prefecture, Japan, the incidence of emergency patients transported by ambulance decreased during the first surge of the COVID-19 pandemic, but the mortality of emergency patients admitted to hospital did not change. The impact of the COVID-19 pandemic on EMS system will need to be monitored over the long term.

\section{Declarations}

\section{Ethics approval and consent to participate}

Because the ORION data is anonymized without specific personal data, such as patient name, date of birth, and address, the requirement of obtaining patient informed consent was waived. The Ethics Committee of Osaka University Graduate School of Medicine approved the waiving of the need of informed consent in this study.

\section{Consent for publication}

Not applicable.

\section{Availability of data and material}

The data that support the findings of this study are available from Osaka Prefectural government but restrictions apply to the availability of these data, which were used under license for the current study, 
and so are not publicly available. Data are however available from the Osaka Prefectural government upon reasonable request and with permission of Osaka Prefectural government.

\section{Competing interests}

None declared.

\section{Funding}

This study was supported by Japanese Society of the Promotion of Science KAKENHI (grant no. JP18H02902).

\section{Author contributions}

YKatayama, TK, SN, MN, SF, TU, YMiyamoto, TB, YMizobata, Yasuyuki Kuwagata, and TM conceived and designed the study. TI and TS supervised the study.

YKatayama, KT, TK, and TT performed and interpreted the statistical analyses. All authors were involved in data acquisition and analysis. YKatayama, KT, and TK prepared the manuscript and figures, with contributions from all authors.

\section{Acknowledgement}

The authors are greatly indebted to all of the EMS personnel and physicians concerned in Osaka Prefecture and to the Osaka Medical Association for their indispensable cooperation and support. We also thank our colleagues from Osaka University Centre of Medical Data Science and Advanced Clinical Epidemiology Investigator's Research Project for providing their insight and expertise for our research.

\section{References}

1 Ministry of Health, Labour and Welfare. The statistics of patients with COVID-19. Available from: https://www.mhlw.go.jp/stf/covid-19/kokunainohasseijoukyou.html\#h2_1. Accessed on 20 November 2020. (in Japanese).

2 Huang C, Wang Y, Li X, et al. Clinical features of patients infected with 2019 novel coronavirus in Wuhan, China. Lancet 2020;395:497-506.

3 Prezant DJ, Zeig-Owens R, Schwartz T, et al. Medical leave associated with COVID-19 among emergency medical system responders and firefighters in New York City. JAMA Netw Open 2020;3:e2016094.

4 Katayama Y, Kitamura T, Kiyohara K, et al. Factors associated with the difficulty in hospital acceptance at the scene by emergency medical service personnel: a population-based study in Osaka City, Japan. BMJ Open 2016;6:e013849. 
5 Okamoto J, Katayama Y, Kitamura T, et al. Profile of the ORION (Osaka emergency information Research Intelligent Operation Network system) between 2015 and 2016 in Osaka, Japan: a populationbased registry of emergency patients with both ambulance and in-hospital records. Acute Med Surg 2019;6:12-24.

6 Takeuchi T, Imanaka T, Katayama Y, Kitamura T, Sobue T, Shimazu T. Profile of patients with novel coronavirus disease 2019 (COVID-19) in Osaka Prefecture, Japan: a population-based descriptive study. $J$ Clin Med 2020;9:2925.

7 Katayama Y, Kiyohara K, Kitamura T, Hayashida S, Shimazu T. Influence of the COVID-19 pandemic on an emergency medical service system: a population-based, descriptive study in Osaka, Japan. Acute Med Surg 2020;7:e534.

8 Osaka Prefectural Government. Estimated population of Osaka Prefecture in 2019. Available from: http://www.pref.osaka.Ig.jp/attach/3387/00014731/R1nenpou.pdf. Accessed on 24 November, 2020 (in Japanese)

9 von Elm E, Altman DG, Egger M, Pocock SJ, Gøtzsche PC, Vandenbroucke JP. The Strengthening the Reporting of Observational Studies in Epidemiology (STROBE) statement: guidelines for reporting observational studies. Lancet 2007;370:1453-7.

10 Ministry of Health, Labour and Welfare. The Treatment Guidelines about COVID-19. Available from: https://www.mhlw.go.jp/content/000631552.pdf. Accessed on 24 November, 2020 (in Japanese)

11 Fire and Disaster Management Agency. The statistics about emergency medical system and rescue activity in 2019. Available from:

https://www.fdma.go.jp/publication/rescue/items/kkkg_r01_01_kyukyu.pdf. Accessed on 24 November, 2020. (in Japanese)

12 Osaka Prefectural Government. Overview of $7^{\text {th }}$ medical plan in Osaka Prefecture. Available from: http://www.pref.osaka.Ig.jp/attach/2502/00281086/compactban\%20kizonbyousyousyuusei.pdf. Accessed on 24 November, 2020 (in Japanese)

13 Katayama Y, Kitamura T, Kiyohara K, et al. Improvements in patient acceptance by hospitals following the introduction of a smartphone app for the emergency medical service system: a populationbased before-and-after observational study in Osaka City, Japan. JMIR Mhealth Uhealth 2017;5:e134.

14 Stella F, Alexopoulos C, Scquizzato T, Zorzi A. Impact of the COVID-19 outbreak on emergency medical system missions and emergency department visits in the Venice area. Eur J Emerg Med 2020;27:298-300.

15 Man CY, Yeung RS, Chung JY, Cameron PA. Impact of SARS on an emergency department in Hong Kong. Emerg Med (Fremantle) 2003;15:418-22. 
$16 \mathrm{Ko}$ PC, Chen WJ, Ma MH, et al. Emergency medical services utilization during an outbreak of severe acute respiratory syndrome (SARS) and the incidence of SARS-associated coronavirus infection among emergency medical technicians. Acad Emerg Med 2004;11:903-11.

17 Tsai MC, Arnold JL, Chuang CC, Chi CH, Liu CC, Yang YJ. Impact of an outbreak of severe acute respiratory syndrome on a hospital in Taiwan, ROC. Emerg Med J 2004;21:311-6.

18 Huang CC, Yen DH, Huang HH, et al. Impact of severe acute respiratory syndrome (SARS) outbreaks on the use of emergency department medical resources. J Chin Med Assoc 2005;68:254-9.

19 Huang $\mathrm{HH}$, Yen DH, Kao WF, Wang LM, Huang Cl, Lee CH. Declining emergency department visits and costs during the severe acute respiratory syndrome (SARS) outbreak. J Formos Med Assoc 2006;105:317.

20 Lapostolle F, Goix L, Vianu I, et al. COVID-19 epidemic in the Seine-Saint-Denis Department of Greater Paris: one month and three waves for a tsunami. Eur J Emerg Med 2020;27:274-8.

21 Saberian P, Conovaloff JL, Vahidi E, Hasani-Sharamin P, Kolivand PH. How the COVID-19 epidemic affected prehospital emergency medical services in Tehran, Iran. West J Emerg Med 2020;21:110-6.

22 Prime Minister's Office of Japan. The Minutes of $15^{\text {th }}$ Headquarters Meeting on COVID-19. Available from: https://www.kantei.go.jp/jp/98_abe/actions/202002/27corona.html. Accessed on 24 November, 2020. (in Japanese)

\section{Figures}




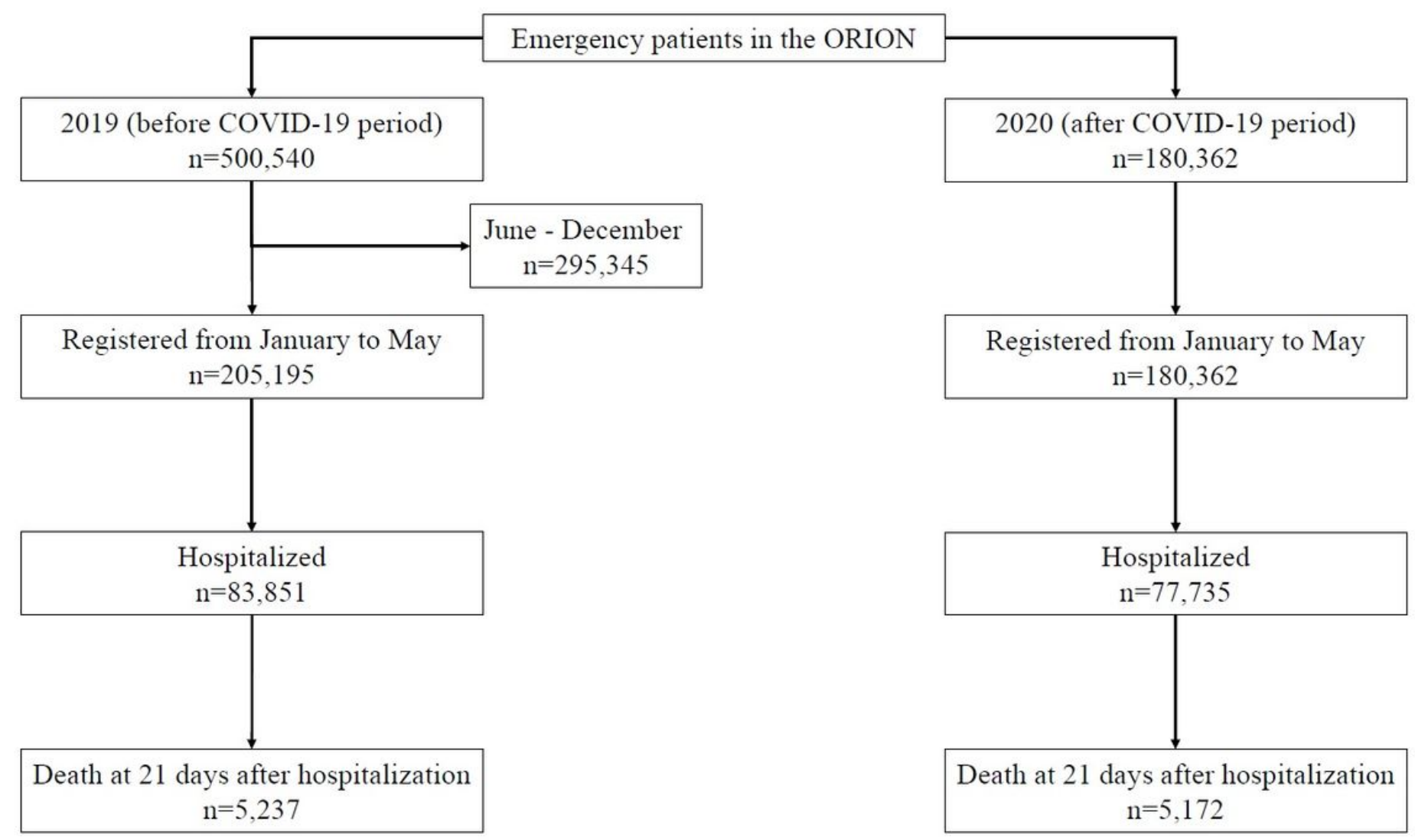

Figure 1

Patient flow in this study. 